\title{
latrogenia grave desconhecida, notificações e notificadores: resultados da actividade da Unidade de Farmacovigilância do Centro
}

Diogo Mendes, ${ }^{*}$ Carlos Alves, ${ }^{* *}$ Francisco Batel Marques***

\section{RESUMO}

Objectivos: Caracterizar as reacções adversas a medicamentos (RAM) notificadas à Unidade de Farmacovigilância do Centro (UFC), documentar o contributo dos profissionais de saúde noticadores de RAM para a farmacovigilância em Portugal e identificar o novo conhecimento produzido decorrente da actividade da UFC.

Tipo de estudo: Observacional, transversal descritivo.

Local: Unidade de Farmacovigilância do Centro.

População: Notificações espontâneas (NE) provenientes da área geográfica correspondente à Administração Regional de Saúde do Centro.

Métodos: Foram incluídas as NE de RAM recebidas na UFC entre Janeiro de 2001 e Agosto de 2011. A causalidade foi imputada de acordo com o método de introspecção global. A gravidade foi atribuída segundo os critérios da Organização Mundial de Saúde (OMS). O conhecimento prévio das RAM foi avaliado segundo a consulta do resumo das características do medicamento (RCM) dos medicamentos suspeitos. Os medicamentos suspeitos foram classificados de acordo com o Prontuário Terapêutico. As RAM foram classificadas de acordo com a terminologia MedDRA.

Resultados: Foram recebidas na UFC 1846 NE de RAM entre 2001 e Agosto de 2011, das quais 999 verificaram os critérios de inclusão. Identificaram-se 149 (14,9\%) RAM desconhecidas e 428 (42,8\%) graves. Destas, 70 (7,0\%) RAM eram simultaneamente desconhecidas e graves e $41(4,1 \%)$ simultaneamente desconhecidas, graves, definitivas ou prováveis. Os médicos notificaram 577 (57,8\%) RAM, das quais $340(34,0 \%)$ por médicos especialistas em medicina geral e familiar. Os farmacêuticos notificaram 354 (35,4\%) RAM e os enfermeiros 66 (6,6\%).

Conclusões: O número de RAM desconhecidas (149; 14,9\%), desconhecidas e graves (70; 7,0\%) e desconhecidas graves definitivas ou prováveis $(41 ; 4,1 \%)$ recebidas na UFC revelam o contributo da NE para o aumento do conhecimento sobre os perfis de segurança dos medicamentos.

Palavras-chave: Farmacovigilância; Sistemas de Notificação de Reacções Adversas a Medicamentos; Reacções Adversas a Medicamentos; Causalidade.

\footnotetext{
* Mestre em Ciências Farmacêuticas. Centro de Avaliação de Tecnologias de Saúde - Unidade de Farmacovigilância do Centro - AIBILI

**Mestre em Ciências Farmacêuticas. Centro de Avaliação de Tecnologias de Saúde - Unidade de Farmacovigilância do Centro - AIBILI; Faculdade de Farmácia da Universidade de Coimbra; Centro de Investigação em Ciências da Saúde - Universidade da Beira Interior

***Licenciado em Ciências Farmacêuticas. Doutor em Ciências Farmacêuticas, na especialidade de Farmácia Clínica. Centro de Avaliação de Tecnologias de Saúde Unidade de Farmacovigilância do Centro - AIBILI; Faculdade de Farmácia da Universidade de Coimbra
}

\section{INTRODUÇÃO}

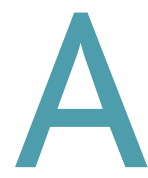
farmacovigilância tem como objectivo a detecção, avaliação e prevenção de reacções adversas a medicamentos (RAM) após a sua comercialização, ${ }^{1,2}$ constituindo uma RAM «qualquer reacção nociva e involuntária a um medicamento que ocorra com doses geralmente utilizadas no ser humano para profilaxia, diagnóstico ou tratamento de doenças ou recuperação, cor- 
recção ou modificação de funções fisiológicas». ${ }^{3}$ A notificação espontânea (NE) é um método da farmacovigilância que permite identificar, documentar e avaliar suspeitas de RAM tendo as vantagens de abranger todos os medicamentos comercializados e todos os doentes, particularmente os que integram populações sub representadas nos ensaios clínicos, para além de poderem detectar RAM raras e/ou de longo tempo de latência. ${ }^{4-6}$

AsRAM reportadas são um contributo insubstituível para o melhor conhecimento da iatrogenia medicamentosa, do perfil de segurança dos medicamentos e para a monitorização contínua das suas relações benefício/risco. ${ }^{6}$

Em Portugal, a Autoridade Nacional do Medicamento e Produtos de Saúde I.P. (INFARMED) é responsável pelo Sistema Nacional de Farmacovigilância (SNF). ${ }^{7}$ O SNF foi criado em 1992 e posteriormente descentralizado em 2000 com a criação das unidades regionais de farmacovigilância (URF). ${ }^{7}$ As suspeitas de RAM são notificadas por profissionais de saúde a uma das quatro unidades de farmacovigilância ou à Direcção de Gestão do Risco de Medicamentos (DGRM) do INFARMED, através do envio de boletins de notificação por correio postal ou electrónico, telefone ou através dos sítios da internet das URF. Os profissionais de saúde podem solicitar por telefone ou $e$-mailà DGRM o envio dos boletins de notificação ou podem imprimir o boletim de notificação disponível no site do INFARMED e enviá-lo à DGRM devidamente preenchido por correio postal. ${ }^{8,9} \mathrm{Em}$ alternativa, os profissionais de saúde podem solicitar também às URF o envio de boletins de notificação ou recorrer aos seus sítios da internet para inserir online os dados relativos às RAM sem necessidade de preencher os boletins em papel. ${ }^{10}$ Os boletins de notificação são ainda distribuídos como documento incluso do Prontuário Terapêutico.

A Unidade de Farmacovigilância do Centro (UFC), sediada na AIBILI (Associação para Investigação Biomédica e Inovação em Luz e Imagem), recebe todas as NE provenientes da área geográfica correspondente à Administração Regional de Saúde do Centro (ARS-C).

Constituíram objectivos deste estudo: caracterizar as RAM notificadas à Unidade de Farmacovigilância do Centro (UFC) entre Janeiro de 2001 e Agosto de 2011 quanto à causalidade imputada, gravidade e conhecimento prévio; documentar o contributo dos profissionais de saúde notificadores de RAM para a farmacovigilância em Portugal; identificar os grupos farmacoterapêuticos com maior prevalência de notificação; e identificar novo conhecimento produzido decorrente da actividade da UFC.

\section{MÉTODOS}

Foi realizado um estudo observacional, transversal, descritivo incluindo as NE provenientes da área geográfica correspondente à ARS-C.

Foram incluídas as NE recebidas na UFC entre Janeiro de 2001 e Agosto de 2011 que verificaram critérios de inclusão primários (identificação de doente, medicamento suspeito, acontecimento adverso e notificador) e secundários (informação completa sobre nível de causalidade imputada, conhecimento prévio avaliado e gravidade documentada).

A codificação das RAM foi feita de acordo com a terminologia MedDRA (Medical Dictionary for Regulatory Activities). A codificação MedDRAéuma terminologia médica específica e padronizada, desenvolvida pela Conferência Internacional de Harmonização (ICH), para facilitar a partilha de informação entre as autoridades reguladoras e a indústria farmacêutica sobre regulamentação de produtos médicos (medicamentos, dispositivos médicos) utilizados por seres humanos. É utilizada para registo, documentação e monitorização da segurança de produtos médicos nas fases de investigação e desenvolvimento e pós-comercialização. ${ }^{11,12}$

Os medicamentos foram classificados de acordo com o Prontuário Terapêutico, porque é um documento de referência extensivamente utilizado pelos profissionais de saúde (médicos, farmacêuticos e enfermeiros) no decorrer das suas actividades profissionais e que segue a Classificação Farmacoterapêutica Portuguesa..$^{13}$

O nível de imputação de causalidade entre a RAM e a exposição ao medicamento suspeito foi classificado segundo os critérios da Organização Mundial da Saúde (OMS), pelo método de introspecção global (IG). ${ }^{14-17} \mathrm{O}$ método da IG é um processo pelo qual um conjunto de peritos avalia a relação causal entre a exposição a um medicamento e a ocorrência de um evento adverso, considerando todos os dados disponíveis relevantes para o caso (dados clínicos do doente, por exemplo). ${ }^{16-18} \mathrm{O}$ grau de probabilidade estabelecido entre a exposição a um medicamento e a ocorrência de determinado evento adverso pode ser definitivo, provável, possível, improvável, condicional/não classificado ou não acessível/não classificável. ${ }^{14-16,19}$ Para se estabelecer uma relação causal deve-se ter em conta os critérios de Bradford Hill: força, consistência, especificidade, temporalidade, gradiente biológico, plausibilidade, coerência, evidência experimental e analogia. ${ }^{16,20} \mathrm{O}$ método da IG é um método simples, de fácil aplicação, que se assemelha ao processo de diagnóstico clínico e que depende dos conhecimentos e da experiência dos peritos. ${ }^{18} \mathrm{O}$ grupo de peritos da UFC é composto por 9 elementos, entre os quais médi- 
cos e farmacêuticos com especializações em farmacologia, farmacologia clínica, farmacovigilância, farmacoepidemiologia, medicina clínica, farmácia clínica e regulamentação farmacêutica e do medicamento. ${ }^{16}$

A gravidade foi atribuída segundo os critérios da OMS. ${ }^{19}$ Foram consideradas graves as RAM que «provocaram morte», «colocaram a vida em risco», «motivaram ou prolongaram a hospitalização», «motivaram incapacidade temporária e/ou definitiva», e que «originaram anomalias médicas».

Foram consideradas conhecidas as RAM descritas nos Resumos das Características dos Medicamentos (RCM) suspeitos.

Os notificadores foram agrupados de acordo com a profissão e local de exercício.

Para o tratamento dos dados foi utilizado o software estatístico SPSS® (Statistical Package for Social Sciences), versão 17.0 .

\section{RESULTADOS}

Foram recebidas na UFC 1846 NE de RAM entre 2001 e Agosto de 2011, 999 das quais verificaram os critérios de inclusão.

O fluxograma do estudo é apresentado na Figura 1. No Quadro I apresenta-se a frequência das RAM distribuída por nível de causalidade imputada, gravidade e conhecimento prévio. Identificaram-se 149 (14,9\%) RAM desconhecidas e 428 (42,8\%) graves. Destas, 70 (7,0\%) RAM eram simultaneamente graves e desconhecidas e $41(4,1 \%)$ simultaneamente graves, desconhecidas, definitivas ou prováveis.

A distribuição do número de notificadores e do número de RAM de acordo com o local de notificação apresenta-se no Quadro II. A consulta em ambulatório foi origem da maior frequência de notificação de RAM (427; 42,7\%). Identificaram-se 423 profissionais de saúde notificadores.

Entre os profissionais de saúde, os médicos $(224 ; 53,0 \%)$ notificaram o maior número de RAM (577; 57,8\%). Os médicos especialistas em medicina geral e familiar (MGF) notificadores $(98 ; 23,2 \%)$ notificaram 340 (34,0\%) RAM.

Os farmacêuticos notificadores (143; 33,8\%) notificaram 354 RAM (35,4\%). A partir de farmácias comunitárias foram notificadas 237 (23,7\%) RAM por 118 (27,8\%) farmacêuticos comunitários. Os farmacêuticos hospitalares $(20 ; 4,7 \%)$ notificaram 112 $(11,2 \%)$ RAM.

Os enfermeiros $(54 ; 12,8 \%)$ notificaram 66 $(6,6 \%)$ RAM. Os enfermeiros notificaram mais frequentemente a partir da consulta em ambulatório (43 RAM; 4,3\%).

A distribuição por grupos terapêuticos dos medicamentos suspeitos descreve-se no Quadro III. Os medicamentos que actuam no «aparelho locomotor» (157; 15,7\%), no «aparelho cardiovascular» (146; 14,6\%), os «anti-infecciosos» $(143 ; 14,3 \%)$ e os que actuam no "sistema nervoso central» (141; $14,1 \%$ ) foram os mais frequentemente notificados. O medicamento que isoladamente foi objecto de maior número de notificações (49; 4,9\%) inclui-se no grupo terapêutico «vacinas e imunoglobulinas».

No Quadro IV apresentam-se as 41 RAM graves, desconhecidas, definitivas ou prováveis distribuídas por grupos terapêuticos. 


\begin{tabular}{|c|c|c|c|c|c|c|c|c|}
\hline \multirow[b]{2}{*}{ Gravidade } & \multicolumn{7}{|c|}{ Nível de imputação de causalidade } & \multirow[b]{2}{*}{ Totais } \\
\hline & $\begin{array}{c}\text { Conhecimento } \\
\text { prévio }\end{array}$ & Definitiva & Provável & Possível & Improvável & $\begin{array}{l}\text { Condicional/ } \\
\text { /Não classificada }\end{array}$ & $\begin{array}{c}\text { Não acessível/ } \\
\text { /Não } \\
\text { classificável }\end{array}$ & \\
\hline Graves & $\begin{array}{l}\text { Conhecidas } \\
\text { Não conhecidas }\end{array}$ & $\begin{array}{r}155 \\
19\end{array}$ & $\begin{array}{l}86 \\
22\end{array}$ & $\begin{array}{l}87 \\
22\end{array}$ & $\begin{array}{l}8 \\
1\end{array}$ & $\begin{array}{l}5 \\
2\end{array}$ & $\begin{array}{r}17 \\
4\end{array}$ & $\begin{array}{r}358 \\
70\end{array}$ \\
\hline \multirow[t]{2}{*}{ Graves } & $\begin{array}{l}\text { Conhecidas } \\
\text { Não conhecidas }\end{array}$ & $\begin{array}{r}130 \\
8\end{array}$ & $\begin{array}{r}180 \\
24\end{array}$ & $\begin{array}{r}124 \\
23\end{array}$ & $\begin{array}{l}9 \\
4\end{array}$ & $\begin{array}{r}17 \\
8\end{array}$ & $\begin{array}{l}32 \\
12\end{array}$ & $\begin{array}{r}492 \\
79\end{array}$ \\
\hline & Totais & 312 & 312 & 256 & 22 & 32 & 65 & 999 \\
\hline
\end{tabular}

Identificaram-se 14 no grupo terapêutico «vacinas e imunoglobulinas».

\section{DISCUSSÃO}

Os profissionais de saúde devem ter em conta os benefícios e os riscos dos medicamentos, bem como a natureza e a severidade das RAM..$^{21-23}$ É reconhecido que o processo da NE contribui para um aumento do conhecimento da relação benefício/risco dos medicamentos. ${ }^{24}$

A UFC encontra-se em actividade há aproximadamente 10 anos. Aolongo desta década recebeu 1846 NE de RAM. Todas estas notificações foram validadas, tratadas e avaliadas na UFC. Desde que iniciou a actividade até à actualidade, a UFC mantém uma base de dados que inclui infor- mações relativas a todas as notificações recebidas. No entanto, verificaram-se 847 notificações que não contêm informação suficiente, nem reparável, para cumprir os requisitos de validação secundária estabelecidos para este trabalho, e foram por isso excluídas. Este achado será objecto de estudo detalhado para identificar possíveis razões para a sua existência, já que tem significativa ponderação relativa no total das notificações recebidas $(45,9 \%)$.

Entre vários objectivos, este estudo investigou qual a importância da UFC na produção de novo conhecimento sobre a segurança dos medicamentos devido à identificação de RAM desconhecidas.

Para identificar o novo conhecimento produzido pela UFC foi necessário caracterizar as RAM quanto a conheci-

\section{QUADRO II. Número de notificadores e RAM por origem das NE.}

\begin{tabular}{|c|c|c|c|c|c|c|c|c|c|c|c|}
\hline \multirow{3}{*}{\multicolumn{2}{|c|}{$\begin{array}{c}\text { Profissional } \\
\text { de Saúde }\end{array}$}} & \multicolumn{8}{|c|}{ Origem da Notificação } & & \\
\hline & & \multicolumn{2}{|c|}{ Ambulatório } & \multicolumn{2}{|l|}{ Hospital } & \multicolumn{2}{|l|}{ Farmácia } & \multicolumn{2}{|l|}{ Outro } & \multicolumn{2}{|l|}{ Totais } \\
\hline & & Notificadores & RAM & Notificadores & RAM & Notificadores & RAM & Notificadores & RAM & Notificadores & RAM \\
\hline \multirow[t]{2}{*}{ Médico } & MGF a) & 98 & 340 & 101 & 185 & - & - & 5c) & 8 & 224 & 577 \\
\hline & Outros & 20 & 44 & & & & & & & & \\
\hline \multicolumn{2}{|c|}{ Farmacêutico } & - & - & 20 & 112 & 118 & 237 & 5c) & 5 & 143 & 354 \\
\hline \multicolumn{2}{|c|}{ Enfermeiro } & 38 & 43 & 16 & 23 & - & - & - & - & 54 & 66 \\
\hline \multicolumn{2}{|l|}{ Outro } & - & - & - & - & $2^{\text {b) }}$ & 2 & - & - & 2 & 2 \\
\hline \multicolumn{2}{|c|}{ Totais RAM } & & 427 & & 320 & & 239 & & 13 & & 999 \\
\hline \multicolumn{2}{|c|}{ Totais Notificadores } & 156 & & 137 & & 120 & & 10 & & 423 & \\
\hline
\end{tabular}

a) médicos de família

b) técnicos de farmácia

c) professores universitários / profissionais em centros de investigação ou indústria 


\begin{tabular}{|lc|}
\hline $\begin{array}{l}\text { QUADRO III. Frequência de RAM por Grupo Terapêutico dos } \\
\text { medicamentos suspeitos. }\end{array}$ \\
\hline Grupo Terapêutico (GT) & RAM (n) \\
\hline Aparelho Locomotor & 157 \\
\hline Aparelho Cardiovascular & 146 \\
\hline Medicamentos Anti-infecciosos & 143 \\
\hline Sistema Nervoso Central & 141 \\
\hline Medicamentos Antineoplásicos e Imunomoduladores & 127 \\
\hline Vacinas e Imunoglobulinas & 122 \\
\hline Outros GT & 163 \\
\hline TOTAL & 999 \\
\hline
\end{tabular}

leccionaram-se estes dois níveis de causalidade por traduzirem uma maior força de associação entre a exposição ao medicamento e a ocorrência dos eventos adversos. Encontraram-se 41 RAM simultaneamente desconhecidas, graves, definitivas ou prováveis que devem merecer especial atenção por parte do Sistema Nacional de Farmacovigilância.

Neste estudo verificou-se que o grupo profissional que mais frequentemente notificou à UFC foi o dos médicos, seguido de farmacêuticos e enfermeiros. A consulta em ambulatório foi o local mais frequente de origem de notificação, seguido por hospital e farmácia comunitária.

No Anuário Estatístico da Região Centro de 2009 contam-se 5861 médicos, dos quais 1361 médicos especialistas em MGF, e 9875 enfermeiros. ${ }^{25}$ De acor-

mento prévio, gravidade e causalidade imputada.

Em primeiro lugar, classificaram-se as RAM quanto ao conhecimento prévio. Foram identificadas 149 RAM desconhecidas, o que revela a importância da farmacovigilância e da NE como fonte de conhecimento de eventos adversos previamente não identificados. Das RAM desconhecidas foram classificadas como graves 70 , cuja documentação é de importância acrescida por representarem eventos adversos que tiveram efeitos clínicos graves. Das RAM desconhecidas graves identificaram-se aquelas cuja causalidade foi imputada como definitiva ou provável. Sedo com os dados fornecidos pela secção regional de Coimbra da Ordem dos Farmacêuticos (OF), até final do ano de 2009 existiam 2049 farmacêuticos (1379 comunitários e 170 hospitalares) registados nesta secção regional. À UFC notificaram, desde 2001 até Agosto de 2011, 224 médicos, dos quais 98 médicos especialistas em MGF, 54 enfermeiros e 143 farmacêuticos (118 comunitários e 20 hospitalares). Com base nestes dados, é possível constatar que os profissionais de saúde notificadores de RAM constituem uma percentagem muito reduzida. Além disso, de acordo com a OMS, um centro de farmacovigilância deverá receber mais
de 200 NE de RAM por milhão de habitantes por ano. ${ }^{26}$ Considerando que a UFC recebeu entre Janeiro de 2001 e Agosto de 2011 um total de 1846 NE de RAM e que a zona centro contava com 2.381.068 habitantes em 2009, ${ }^{25}$ concluímos que o número de NE de RAM enviadas à UFC é baixo, ilustrando que a sub-notificação continua a ser uma limitação da farmacovigilância. Apesar das limitações descritas, foi possível identificar 41 RAM simultaneamente desconhecidas, graves, definitivas ou prováveis. Se o número de NE aumentar, nomeadamente através de uma maior adesão dos profissionais de saúde a este método de monitorização da segurança dos medicamentos na prática clínica, será 
possível produzir mais e melhor conhecimento cuja consequência será a melhor avaliação das relações benefício/risco dos medicamentos e a maior segurança dos doentes.

A NE constitui um processo simples e não dispendioso que permite aos profissionais de saúde contribuírem para melhorar o conhecimento do perfil de segurança dos medicamentos. No entanto, a falta de informação sobre o SNF, em particular sobre o processo de NE de RAM, bem como a dificuldade em reconhecer um evento adverso como consequência da exposição a um medicamento, são factores que podem ajudar a explicar a muito reduzida percentagem de notificadores encontrada. A promoção de acções formativas e de divulgação sobre o SNF junto dos profissionais de saúde e a integração deste tema na formação académica poderá contribuir para a melhoria do sistema de farmacovigilância. Por outro lado, os profissionais de saúde, enquanto notificadores, devem estar conscientes de que notificar suspeitas de RAM é um dever profissional.

Este estudo permitiu também identificar os grupos terapêuticos com maior prevalência de notificação. Os medicamentos que actuam no «aparelho locomotor», no «aparelho cardiovascular», os «anti-infecciosos» e os que actuam no «sistema nervoso central» representaram $60 \%$ dos medicamentos notificados como suspeitos. No entanto, a ausência de dados de exposição populacional a estes medicamentos não permite tirar conclusões definitivas nem generalizar os resultados obtidos.

Em conclusão, apesar das limitações referidas, é demonstrado que a NE é um método valioso na detecção de RAM raras e graves. ${ }^{5,6}$

Nota: a UFC exerceu a sua actividade entre 2001 e 2004 e de 2009 até ao presente.

\section{AGRADECIMENTOS}

A todos os profissionais de saúde notificadores da zona geográfica correspondente à Administração Regional de Saúde do Centro.

À secção regional de Coimbra da Ordem dos Farmacêuticos pelos dados gentilmente cedidos.

\section{REFERÊNCIAS BIBLIOGRÁFICAS}

1. European Commission. Volume 9A of the Rules Governing Medicinal Products in the European Union - Guidelines on Pharmacovigilance for Medicinal Products for Human Use. Final September 2008.

2. Mann RD, Andrews EB, editors. Pharmacovigilance. 2nd ed. Chichester: John Wiley \& Sons; 2007.

3. Decreto-Lei n. ${ }^{\circ}$ 176/2006, de 30 Agosto. Diário da República, n. ${ }^{\circ} 167$, Série I. Estatuto do Medicamento. Disponível em http://www.dre.pt/ pdf1s/2006/08/16700/62976383.pdf [acedido em 12/09/2011].

4. Meyboom RH, Hekster YA, Egberts AC, Gribnau FW, Edwards IR. Causal or casual? The role of causality assessment in pharmacovigilance. Drug Saf 1997 Dec; 17 (6): 374-89.

5. Goldman SA. Limitations and strengths of spontaneous reports data. Clin Ther 1998; 20 Suppl C: C40-4.

6. Hartmann K, Doser AK, Kuhn M. Postmarketing safety information: how useful are spontaneous reports? Pharmacoepidemiol Drug Saf 1999 Apr; 8 Suppl 1: S65-71.

7. Decreto-Lei n. ${ }^{\circ}$ 242/2002, de 05 de Novembro de 2002. Diário da República, n. ${ }^{\circ} 255$, I Série-A. Regulamento do Sistema Nacional de Farmacovigilância. Disponível em: http://dre.pt/pdf1sdip/2002/ 11/255A00/70867091.pdf [acedido em 12/09/2011].

8. INFARMED. Medicamentos de Uso Humano. Farmacovigilância. Notificação de RAM. Disponível em: http://www.infarmed.pt/portal/ page/portal/INFARMED/MEDICAMENTOS_USO_HUMANO/FARMACOVIGILANCIA/NOTIFICACAO_DE_RAM [acedido em 31/10/2011].

9. INFARMED. Medicamentos de Uso Humano. Farmacovigilância. Ficha de Notificação de RAM. Disponível em: http://www.infarmed.pt/ portal/page/portal/INFARMED/MEDICAMENTOS_USO_HUMANO/FA RMACOVIGILANCIA/NOTIFICACAO_DE_RAM/ficha_notificacao.pdf [acedido em 31/10/2011].

10. Sítios da Internet para notificação de RAM. Unidade Regional de Farmacovigilância do Norte em http://ufn.med.up.pt/; Unidade Regional de Farmacovigilância do Centro em http://www.ufc.aibili.pt/; Unidade Regional de Farmacovigilância do Sul em http://ufs.ff.ul.pt/Notificacao.aspx; Unidade de Farmacovigilância de Lisboa eVale do Tejo através do e-mail uflvt@sapo.pt [acedido em 31/10/2011].

11. International Conference on Harmonisation of Technical Requirements for Registration of Pharmaceuticals for Human Use (ICH). Medical Dictionary for Regulatory Activities (MedDRA). Disponível em: http://www. ich.org/products/meddra.html [acedido em 31/10/2011].

12. Maintenance and Support Services Organization (MSSO). Medical Dictionary for Regulatory Activities (MedDRA). Disponível em: http://www. meddramsso.com/ [acedido em 31/10/2011].

13. Despacho n. ${ }^{\circ} 21844 / 2004$, de 12 de Outubro. Diário da República, 2. ${ }^{a}$ série, n. ${ }^{\circ} 131$, de 6 de Junho de 2004. Homologa a classificação farmacoterapêutica de medicamentos. Disponível em: http://www.infarmed.pt/portal/page/portal/INFARMED/LEGISLACAO/LEGISLACAO_ FARMACEUTICA_COMPILADA/TITULO_IV/despacho_21884_2004.pdf [acedido em 12/09/2011].

14. World Health Organization (WHO), Uppsala Monitoring Centre. The use of the WHO-UMC system for standardised case causality assessment. Disponível em: http://who-umc.org/Graphics/24734.pdf [acedido em 12/09/2011].

15. Macedo AF, Alves C, Craveiro N, Batel-Marques F. A exposição simultânea a vários medicamentos como factor de risco de reacções adversas graves. Rev Port Farmacoter 2010; 2: 163-6.

16. Craveiro N, Alves C, Batel-Marques F. Níveis de causalidade imputados a reacções adversas a medicamentos em função do profissional notificador: potencial influência do acesso à informação clínica. Rev Port Farmacoter 2010; 2: 89-93.

17. Macedo AF, Marques FB, Ribeiro CF, Teixeira F. Causality assessment of adverse drug reactions: comparison of the results obtained from published decisional algorithms and from the evaluations of an expert panel, according to different levels of imputability. J Clin Pharm Ther 
2003 Apr; 28 (2): 137-43.

18. Agbabiaka TB, Savovíc J, Ernst E. Methods for causality assessment of adverse drug reactions: a systematic review. Drug Saf 2008; 31 (1): 2137.

19. The Uppsala Monitoring Centre. World Health Organization. Safety Monitoring of Medicinal Products - Guidelines for setting up and running a Pharmacovigilance Centre. The Uppsala Monitoring Centre (the UMC), WHO Collaborating Centre for International Drug Monitoring. Uppsala, Sweden. 2000. Disponível em: http://apps.who.int/medicinedocs/en/d/Jh2934e/ [acedido em 31/10/2011].

20. Hill BA. The environment and disease: Association or causation? Proc R Soc Med 1965 May; 58: 295-300.

21. Moore N, Lecointre D, Noblet C, Mabille M. Frequency and cost of serious adverse drug reactions in a department of general medicine. $\mathrm{Br}$ J Clin Pharmacol 1998 Mar; 45 (3): 301-8.

22. Koh Y, Kutty FB, Li SC. Drug-related problems in hospitalized patients on polypharmacy: the influence of age and gender. Ther Clin Risk Manag 2005 Mar; 1 (1): 39-48.

23. Loke YK, Derry S. Reporting of adverse drug reactions in randomised controlled trials - a systematic survey. BMC Clin Pharmacol. 2001; 1:3.

24. Rossi AC, Knapp DE. Discovery of new adverse drug reactions. A review of the Food and Drug Administration's spontaneous reporting system. JAMA 1984 Aug 24-31; 252 (8): 1030-3.
25. Anuário Estatístico da Região Centro 2009. Instituto Nacional de Estatística, I.P. Lisboa, Portugal. Disponível em: http://www.ine.pt/xportal/xmain?xpid=INE\&xpgid=ine_publicacoes\&PUBLICACOESpub_boui $=102943595 \&$ PUBLICACOESmodo $=2$ [acedido em 31/10/2011].

26. The Uppsala Monitoring Centre. World Health Organization. Pharmacovigilance - Reporting Trends. Disponível em: http://who-umc.org/ DynPage.aspx id $=108476 \& \mathrm{mn} 1=7347 \& \mathrm{mn} 2=7252 \& \mathrm{mn} 3=7322 \& \mathrm{~m}$ n4=7558 [acedido em 28/09/2011].

\section{CONFLITOS DE INTERESSE}

Os autores declaram não possuir qualquer tipo de conflitos de interesse.

\author{
ENDEREÇO PARA CORRESPONDÊNCIA \\ Diogo Mendes \\ Unidade de Farmacovigilância do Centro \\ AIBILI \\ Azinhaga de Santa Comba - Celas \\ 3000-548 Coimbra \\ Portugal \\ e-mail: diogomendes26@gmail.com
}

Recebido em 04/10/2011

Aceite para publicação em 13/11/2011

\section{ABSTRACT}

\section{SEVERE IATROGENIC ADVERSE DRUG REACTION REPORTS: RESULTS OF THE ACTIVITY OF THE REGIONAL PHARMACOVIGILANCE UNIT OF CENTRAL PORTUGAL}

Objectives: This study aims to characterize the adverse drug reactions (ADR) reported to the Regional Pharmacovigilance Unit of Central Portugal (UFC), to document the contribution of reports of ADR by health professionals to pharmacovigilance in Portugal, and to identify the new knowledge produced by the activity of the UFC.

Type of study: Cross-sectional.

Setting: Regional Pharmacovigilance Unit of Central Portugal.

Population: Spontaneous reports from the geographic area corresponding to the Health Administration of the Central Region of Portugal.

Methods: Spontaneous reports (SR) of ADR received in the UFC between January 2001 and August 2011 were included. Causality was assessed using the global introspection method. Severity was assessed according to World Health Organization (WHO) criteria. Previous description of an ADR was assessed by consulting the Summary of Product Characteristics. Suspected drugs were classified in categories according to the Portuguese Drug and Therapeutics Bulletin. Suspected drugs were classified in categories according to the Bulletin. ADR were classified according to the MedDRA dictionary.

Results: The UFC received 1846 SR of ADR between 2001 and August 2011.999 met the inclusion criteria. We identified 149 (14.9\%) unknown ADR and $428(42.8 \%)$ serious ADR. 70 (7.0\%) ADR were simultaneously unknown and serious and 41 (4.1\%) simultaneously unknown, serious, and certain or probable. Physicians reported 577 (57.8\%) ADR, of which 340 (34.0\%) were reported by family physicians. Pharmacists reported 354 (35.4\%) ADR and nurses 66 (6.6\%).

Conclusions: Unknown ADR (149; 14.9\%), unknown and serious ADR (70; 7.0\%) and unknown serious certain or probable ADR (41; $4.1 \%)$ received by the UFC show that SR are of great value in increasing knowledge about the safety profiles of drugs.

Keywords: Drug Surveillance, Postmarketing; Adverse Drug Reaction Reporting Systems; Adverse effects; Causality. 